\title{
Auf dem Weg zur Eliminationsgesellschaft?
}

\section{Peter Weibel}

Dr. med., Geriater und Schriftsteller

Eine klare Mehrheit der Schweizer Bevölkerung würde die Möglichkeit eines erleichterten Alterssuizids begrüssen. (Zeitung Reformiert, 10. Oktober 2014)

- Der Begriff Alterssuizid verbindet Alter mit Suizid. Zum Gebrauchswort geworden, verfestigt er die Nähe von Alter und Suizidwilligkeit.

- Jedes Gebrauchswort schafft sich seine eigene Wirklichkeit.

- Die Wirklichkeit des erleichterten Alterssuizids (erleichterter Zugang zum Sterbemedikament) stützt sich auf das Diktat der Selbstbestimmung des aufgeklärten Menschen. Der Begriff Selbstbestimmung wird dazu gebraucht und missbraucht.

- Erleichterter Alterssuizid bedeutet nicht selbstbestimmte Lebensbeendigung aufgrund einer ausweglosen Krankheit, sondern aufgrund eines hohen biologischen Alters und der Einschränkungen des Alters.

- Die Bedeutung der Selbstbestimmung in der Gesellschaft heute ist unwiderlegbar, aber sie ist auch gefährlich. Sie tritt an die Stelle der Gottgläubigkeit, wo Gott für viele Menschen schweigt. Sie ist gefährlich, weil das Selbst immer auch in Bezug zu anderen Menschen steht, und weil die innere Bestimmung nie frei von äusserem Bestimmenden ist.

- Freiverantwortlichkeit setzt die Freiheit des Entscheidens und Handelns voraus, und sie entbindet die Umwelt von der Mitverantwortung.

- Die Freiheit, selbst zu bestimmen, kann auch die Unfreiheit sein, durch äussere Not oder äussere Wertbestimmung keine andere Wahl mehr zu haben.

- Das innere Wertbild des Menschen ist immer auch ein Spiegel des äusseren.

- Wenn die Gesellschaft oder Teile der Gesellschaft die Bedingungen umreissen, unter denen Leben nicht mehr lebbar sein könnte, werden auch die Wertbedingungen des Lebens festgelegt.

- Wertbedingungen sind Signale zur Eingrenzung und Ausgrenzung von Leben, wobei das Wort sinnvoll gebraucht wird. Aber die Sinnfrage endet nicht, sie beginnt erst, wenn sie geklärt scheint.

- Die Sinnfrage liegt nicht nur im Leiden selbst, sie liegt auch in der Antwort auf das Leiden.
- Sterbehilfeorganisationen halten am Feindbild einer ungebremsten Medizin fest. Sie weisen die Tatsache ab, dass die Altersmedizin heute nicht mehr vom Machbaren, aber vom Ertragbaren und Wünschbaren geleitet wird. Eine medizinische Lebenskultur und eine palliative Sterbekultur sind keine Gegensätze, es sind Ergänzungen.

- Wertbedingungen können zu gesellschaftlichen Richtlinien werden. Richtlinien für versehrte Menschen, von Unversehrten festgelegt, Richtlinien für sehr alte Menschen, von Noch-nicht-Alten festgelegt.

- Wertbestimmungen werden die Zukunft des Menschen elementar verändern.

- Sie tragen das eigene Angstbild einer Abhängigkeit ohne Würde, und sie zementieren das Angstbild beim abhängigen alten Menschen.

- Der zementierte Zweifel, dass es eine Abhängigkeit ohne Würde nicht geben kann, nimmt - zu Ende gedacht - die Möglichkeit, der Abhängigkeit Würde zu geben.

- Die Beschleunigung am Lebensende weist die Chance $a b$, eine Würdelosigkeit nicht hinzunehmen, und sie bricht mit der Kultur des Abschieds und des Warten-Könnens.

- Der Verlust von Abschiedlichkeit und der Fähigkeit, warten zu können, ist eine Entfernung von menschlichen Grundwerten.

- Die Beschleunigung am Lebensende ist nicht unabhängig von der Beschleunigung, mit der sich die materielle Welt vorantreibt.

- Dass sie der materiellen Welt auch dient, ist nur logisch.

- Die Entsorgung von ohnmächtig machendem Altersleiden - und der Option Widerstand gegen das Leiden - wird die Gesellschaft und den Menschen in der Gesellschaft verändern.

- Die Gesellschaft wird dadurch nicht menschlicher werden.

- Die Welt wird nicht vollkommener, wenn sie das verstörend Unvollkommene abweist.

- Die Antworten, die in die Mitte des Lebens führen, kommen immer von den Rändern des Lebens.

- Teil der Antwort liegt nicht im Prinzip Lösung, aber im Nicht-Hinnehmen des Unlösbaren. 